\title{
Effect of Size, Coverage, and Dispersity on the Potential-Controlled Ostwald Ripening of Metal Nanoparticles
}

Dhruba K. Pattadar and Francis P. Zamborini*

Department of Chemistry, University of Louisville, Louisville, Kentucky 40292, United States

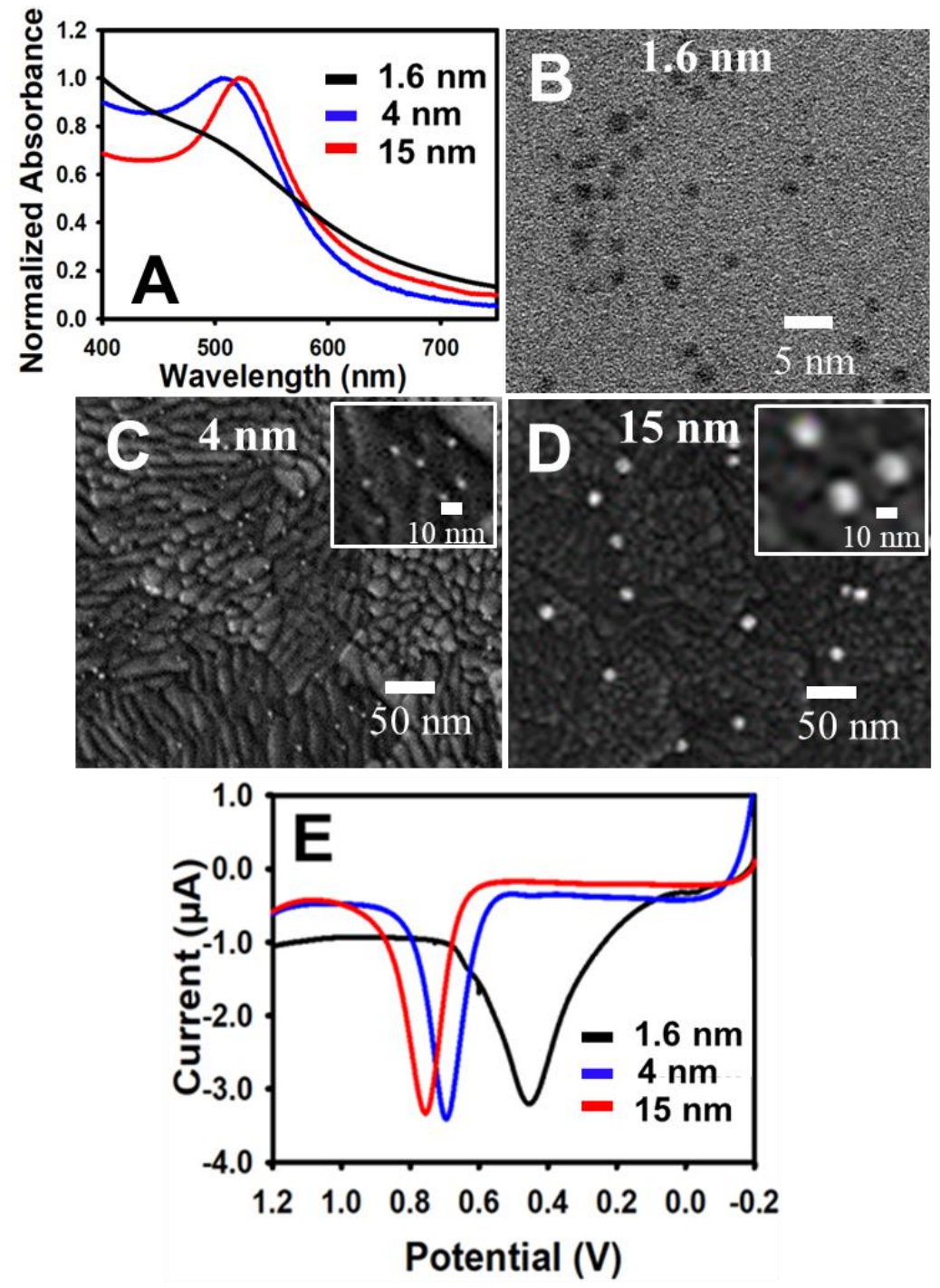

Figure S1. (A) UV-Vis spectra of THPC-stabilized $1.6 \mathrm{~nm}$ and citrate-coated 4 and $15 \mathrm{~nm}$ diameter Au NPs. (B) TEM image of THPC-stabilized $1.6 \mathrm{~nm}$ Au NPs. (C, D) SEM images of 4 and $15 \mathrm{~nm}$ citrate-stabilized Au NPs. (E) ASV of 1.6, 4, and $15 \mathrm{~nm}$ diameter Au NPs. ASVs were obtained in $10 \mathrm{mM} \mathrm{KBr}$ plus $0.1 \mathrm{M} \mathrm{KClO}_{4}$ electrolyte at a scan rate of $0.01 \mathrm{~V} / \mathrm{s}$. 

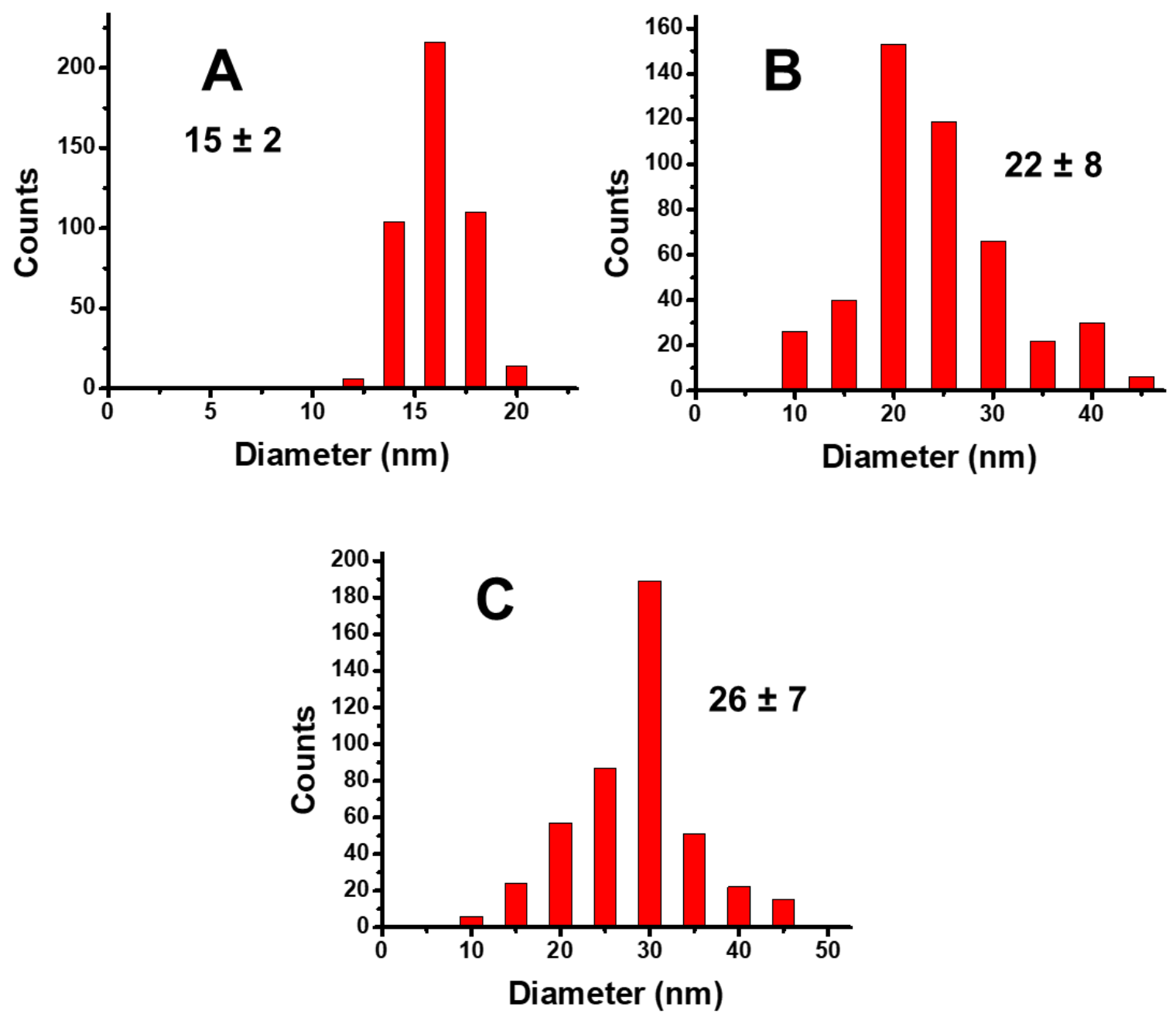

Figure S2. Size histogram of $15 \mathrm{~nm}$ Au NPs (A) without Ostwald ripening, (B) after $140 \mathrm{~min}$ of ripening at $0.30 \mathrm{~V}$ and $(\mathrm{C})$ after $140 \mathrm{~min}$ of ripening at $0.45 \mathrm{~V}$. 

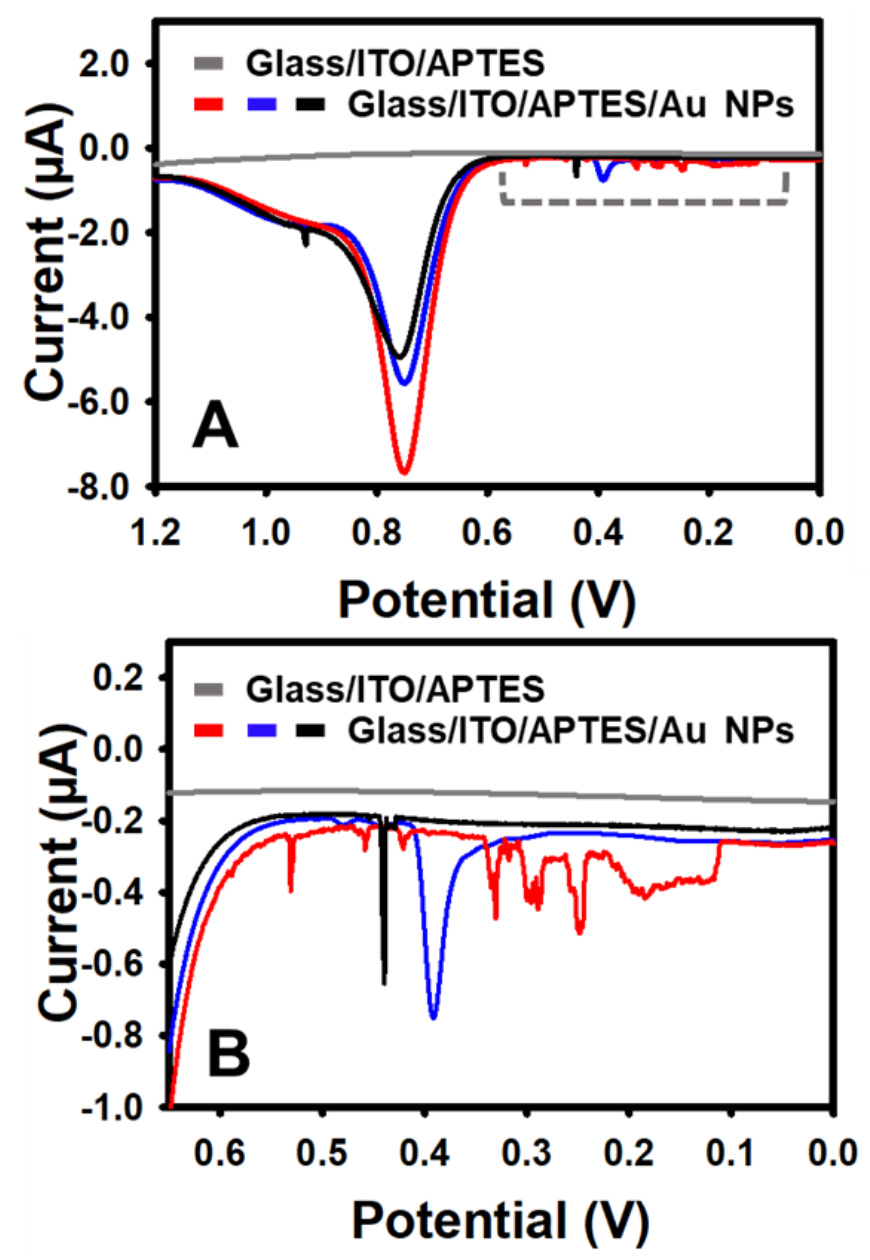

Figure S3. ASV of citrate-stabilized $15 \mathrm{~nm}$ diameter Au NPs after $105 \mathrm{~min}$ of Ostwald ripening at $0.30 \mathrm{~V}(\mathrm{~A})$. ASV obtained in $10 \mathrm{mM} \mathrm{KBr}$ plus $0.1 \mathrm{M} \mathrm{KClO}_{4}$ electrolyte at a scan rate of 0.01 $\mathrm{V} / \mathrm{s}$. (B) The zoomed in portion of Figure $\mathrm{A}$ as indicated by the dashed rectangle. 

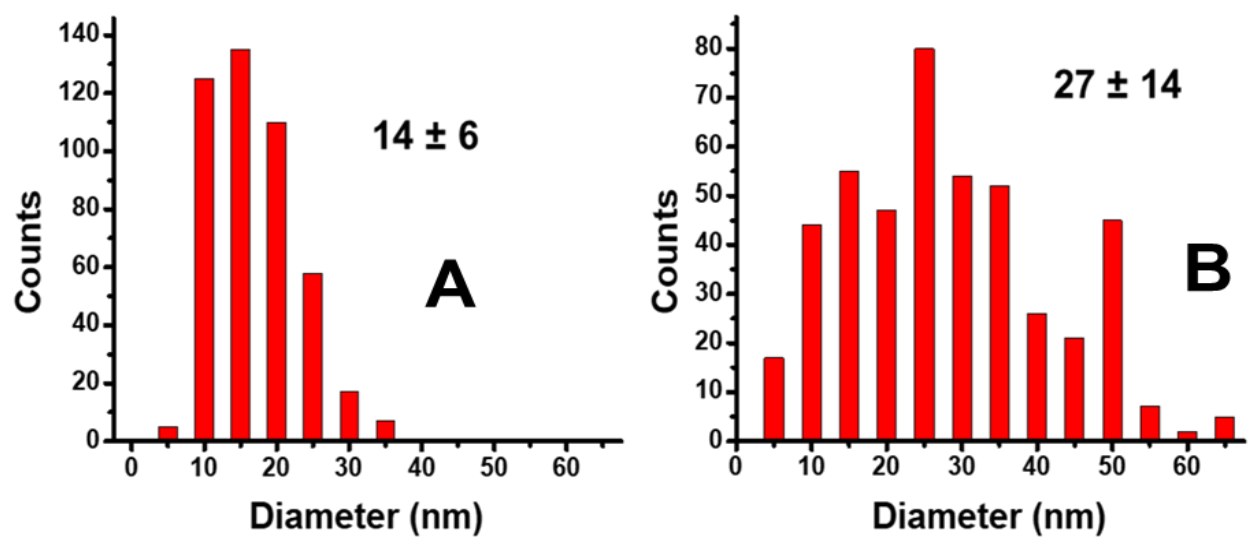

Figure S4. Size histogram of $4 \mathrm{~nm} \mathrm{Au} \mathrm{NPs} \mathrm{after} 140 \mathrm{~min}$ of ripening at $0.30 \mathrm{~V}$ (A) and after 140 min of ripening at $0.45 \mathrm{~V}$ (B).
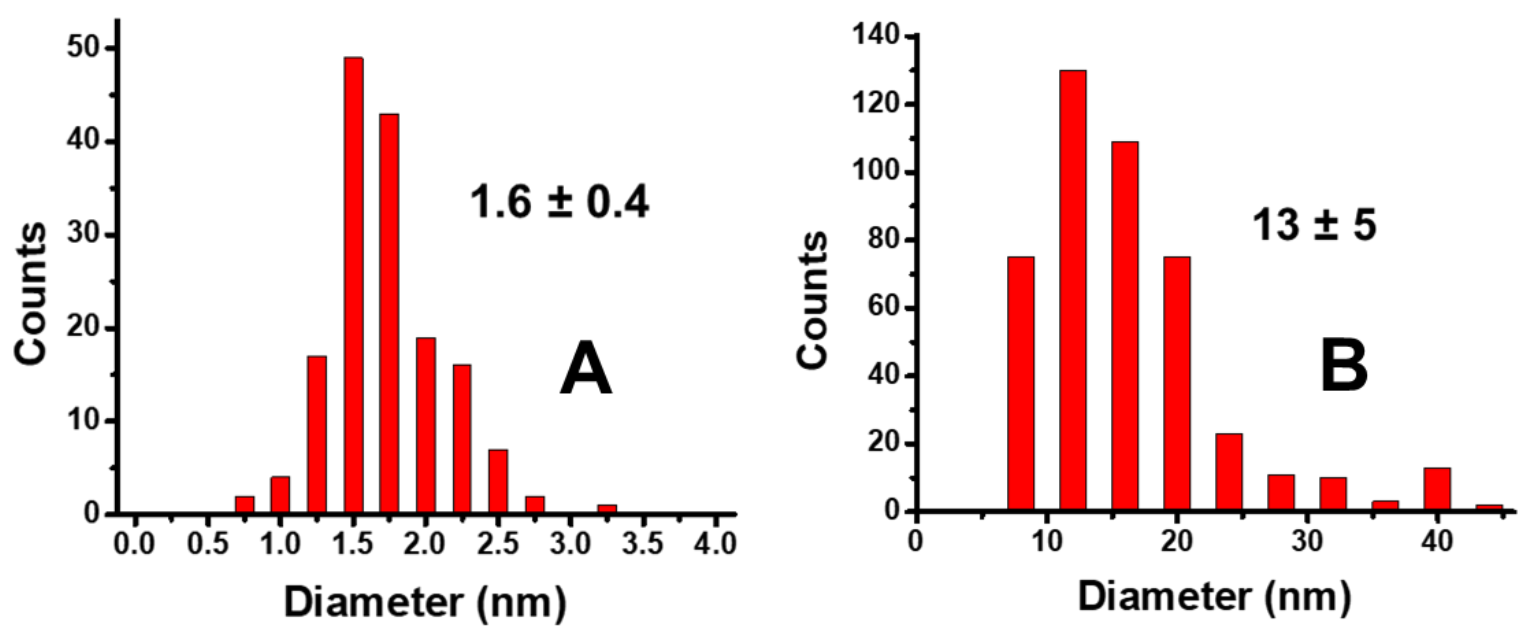

Figure S5. Size histogram of $1.6 \mathrm{~nm}$ diameter Au NPs before treatment (A) and after $140 \mathrm{~min}$ of ripening at $0.30 \mathrm{~V}(\mathrm{~B})$. 


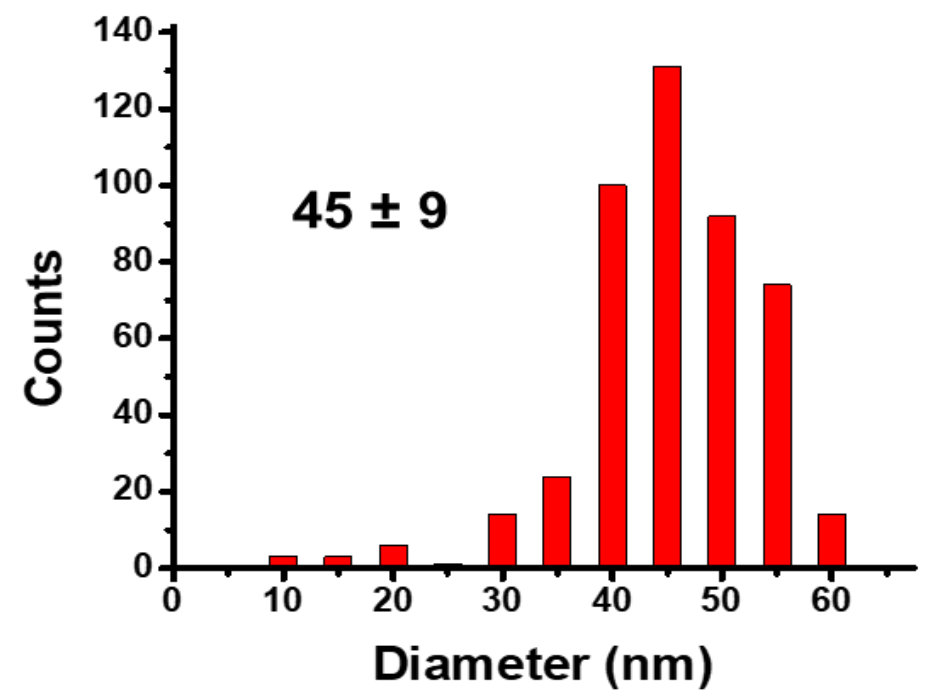

Figure S6. Size histogram of 1:3 mixture of 4:15 nm Au NPs after $140 \mathrm{~min}$ of ripening at $0.30 \mathrm{~V}$. 

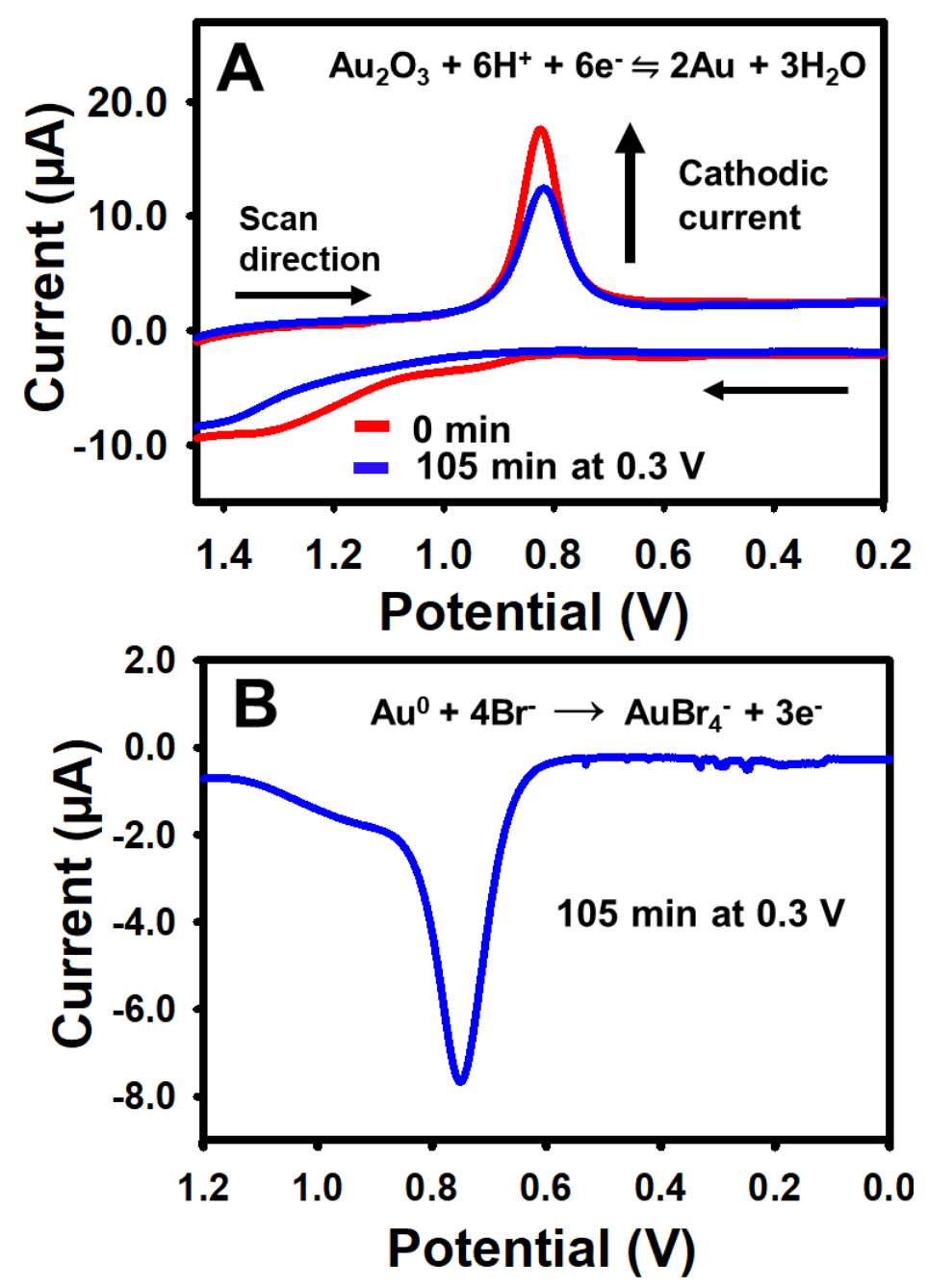

Figure S7. (A) Cyclic voltammogram (CV) of $15 \mathrm{~nm}$ Au NPs before (0 min) and after 105 min of holding at $0.30 \mathrm{~V}$ vs $\mathrm{Ag} / \mathrm{AgCl}$ in $10 \mathrm{mM} \mathrm{KBr}$ plus $0.1 \mathrm{M} \mathrm{HClO}_{4}$ electrolyte solution. (B) ASV of the same electrode after $105 \mathrm{~min}$ of ripening at $0.30 \mathrm{~V}$. ASV was performed in 10 $\mathrm{mM} \mathrm{KBr}$ plus $0.1 \mathrm{M} \mathrm{KClO}_{4}$ solution. The $\mathrm{CV}$ coverage (electroactive surface area, SA) decreases from $7.65 \times 10^{-6} \mathrm{C}$ to $5.76 \times 10^{-6} \mathrm{C}$ and the ASV coverage (total volume of NPs, V) $3.96 \times 10^{-5} \mathrm{C}$. The SA/V values before and after ripening were 0.19 and 0.14 , which corresponds to 12.0 and $19.4 \mathrm{~nm}$ diameter $\mathrm{Au} \mathrm{NPs}$, respectively. 
Table S1. Microscopy measured radius and electrochemically measured surface area-to-total volume ratio (SA/V) of different sized Au NPs.

\begin{tabular}{|c|c|c|}
\hline $\begin{array}{c}\text { Number of } \\
\text { trials }\end{array}$ & $\begin{array}{c}\text { Microscopy } \\
\text { measured } \\
\text { radius (nm) }\end{array}$ & $\begin{array}{c}\text { Average } \\
\text { SA/V }\end{array}$ \\
\hline 4 & $0.75 \pm 0.2$ & $2.34 \pm 0.07$ \\
\hline 4 & $2.0 \pm 0.4$ & $0.64 \pm 0.03$ \\
\hline 4 & $7.5 \pm 0.8$ & $0.23 \pm 0.08$ \\
\hline 4 & $15.6 \pm 0.9$ & $0.10 \pm 0.01$ \\
\hline 4 & $25.1 \pm 1.5$ & $0.06 \pm 0.01$ \\
\hline 4 & $35.4 \pm 1.4$ & $0.04 \pm 0.01$ \\
\hline
\end{tabular}

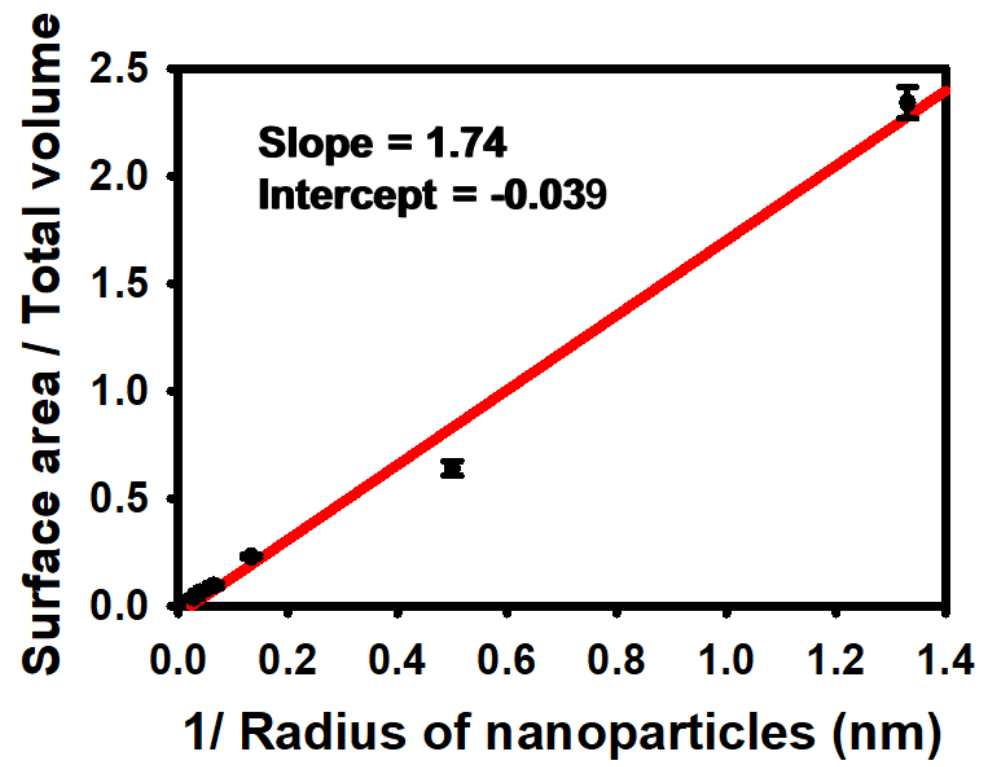

Figure S8. Plot of electrochemically-measured surface area-to-total volume ratio (SA/V) as a function of 1/radius as measured by TEM or SEM for 1.6, 4, 15, 31, 50, and $70 \mathrm{~nm}$ diameter Au NPs. The surface area was measured by surface $\mathrm{Au}$ oxide formation and reduction in $0.1 \mathrm{M} \mathrm{HClO}_{4}$ and the total volume was measured by complete Au stripping in $10 \mathrm{mM} \mathrm{KBr}$ plus $0.1 \mathrm{M} \mathrm{KClO}_{4}$ electrolyte. 
Table S2. SA/V for $15 \mathrm{~nm}$ diameter Au NPs before and after ripening at $0.0 \mathrm{~V}$.

\begin{tabular}{|c|c|c|c|c|c|c|c|}
\hline $\begin{array}{c}\text { Trial } \\
\#\end{array}$ & $\begin{array}{l}\text { Time of } \\
\text { Ostwald } \\
\text { ripening } \\
\text { (min) }\end{array}$ & $\begin{array}{c}\text { Au oxide } \\
\text { reduction } \\
\text { charge in } \mathrm{CV} \\
\text { (before Ost.) } \\
\text { (C) }\end{array}$ & $\begin{array}{c}\text { Au oxide } \\
\text { reduction } \\
\text { charge in CV } \\
\text { (after Ost.) } \\
\text { (C) }\end{array}$ & $\begin{array}{c}\text { Au stripping } \\
\text { charge in } \\
\text { ASV } \\
\text { (total volume) } \\
\text { (C) }\end{array}$ & $\begin{array}{c}\text { Surface } \\
\text { area (SA)/ } \\
\text { Volume } \\
\text { (after Ost.) } \\
\text { (V) }\end{array}$ & $\begin{array}{l}\text { Calculated } \\
\text { NP } \\
\text { diameter } \\
\text { based } \\
\text { on cal. } \\
\text { curve (nm) }\end{array}$ & $\begin{array}{c}\text { Average } \\
\text { diameter } \\
\text { of NPs } \\
\text { based on } \\
\text { cal. curve } \\
\text { (nm) }\end{array}$ \\
\hline 1 & 0 & $9.46 \times 10^{-6}$ & & $3.80 \times 10^{-5}$ & 0.248 & 12.1 & \multirow{4}{*}{$12.6 \pm 0.5$} \\
\hline 2 & 0 & $7.67 \times 10^{-6}$ & & $3.42 \times 10^{-5}$ & 0.224 & 13.2 & \\
\hline 3 & 0 & $1.02 \times 10^{-5}$ & & $4.39 \times 10^{-5}$ & 0.232 & 12.8 & \\
\hline 4 & 0 & $1.05 \times 10^{-5}$ & & $4.25 \times 10^{-5}$ & 0.247 & 12.1 & \\
\hline 1 & 35 & $7.91 \times 10^{-6}$ & $7.15 \times 10^{-6}$ & $3.76 \times 10^{-5}$ & 0.190 & 15.2 & \multirow{4}{*}{$13.7 \pm 1.3$} \\
\hline 2 & 35 & $7.56 \times 10^{-6}$ & $6.75 \times 10^{-6}$ & $2.82 \times 10^{-5}$ & 0.239 & 12.5 & \\
\hline 3 & 35 & $6.57 \times 10^{-6}$ & $6.38 \times 10^{-6}$ & $3.18 \times 10^{-5}$ & 0.201 & 14.5 & \\
\hline 4 & 35 & $8.08 \times 10^{-6}$ & $7.54 \times 10^{-6}$ & $3.24 \times 10^{-5}$ & 0.232 & 12.8 & \\
\hline 1 & 70 & $9.61 \times 10^{-6}$ & $7.20 \times 10^{-6}$ & $3.58 \times 10^{-5}$ & 0.201 & 14.5 & \multirow{4}{*}{$14.4 \pm 0.2$} \\
\hline 2 & 70 & $9.35 \times 10^{-6}$ & $7.84 \times 10^{-6}$ & $3.86 \times 10^{-5}$ & 0.204 & 14.3 & \\
\hline 3 & 70 & $9.48 \times 10^{-6}$ & $7.73 \times 10^{-6}$ & $3.88 \times 10^{-5}$ & 0.199 & 14.6 & \\
\hline 4 & 70 & $7.67 \times 10^{-6}$ & $5.91 \times 10^{-6}$ & $2.84 \times 10^{-5}$ & 0.208 & 14.1 & \\
\hline 1 & 105 & $9.64 \times 10^{-6}$ & $7.13 \times 10^{-6}$ & $3.57 \times 10^{-5}$ & 0.199 & 14.6 & \multirow{4}{*}{$14.7 \pm 0.3$} \\
\hline 2 & 105 & $8.21 \times 10^{-6}$ & $7.20 \times 10^{-6}$ & $3.60 \times 10^{-5}$ & 0.200 & 14.5 & \\
\hline 3 & 105 & $7.65 \times 10^{-6}$ & $6.19 \times 10^{-6}$ & $3.25 \times 10^{-5}$ & 0.190 & 15.2 & \\
\hline 4 & 105 & $7.20 \times 10^{-6}$ & $6.51 \times 10^{-6}$ & $3.25 \times 10^{-5}$ & 0.200 & 14.5 & \\
\hline 1 & 140 & $7.37 \times 10^{-6}$ & $6.23 \times 10^{-6}$ & $3.27 \times 10^{-5}$ & 0.191 & 15.1 & \multirow{4}{*}{$14.8 \pm 0.5$} \\
\hline 2 & 140 & $9.38 \times 10^{-6}$ & $7.01 \times 10^{-6}$ & $3.73 \times 10^{-5}$ & 0.188 & 15.3 & \\
\hline 3 & 140 & $7.81 \times 10^{-6}$ & $7.10 \times 10^{-6}$ & $3.45 \times 10^{-5}$ & 0.206 & 14.2 & \\
\hline 4 & 140 & $9.12 \times 10^{-6}$ & $6.30 \times 10^{-6}$ & $3.18 \times 10^{-5}$ & 0.198 & 14.7 & \\
\hline
\end{tabular}


Table S3. SA/V ratio for $15 \mathrm{~nm}$ diameter Au NPs before and after ripening at $0.30 \mathrm{~V}$.

\begin{tabular}{|c|c|c|c|c|c|c|c|}
\hline $\begin{array}{l}\text { No. } \\
\text { of } \\
\text { trial }\end{array}$ & $\begin{array}{c}\text { Time of } \\
\text { Ostwald } \\
\text { ripping } \\
\text { (min) }\end{array}$ & $\begin{array}{c}\text { Au oxide } \\
\text { reduction } \\
\text { charge in } \mathrm{CV} \\
\text { (before Ost.) } \\
\text { (C) }\end{array}$ & $\begin{array}{l}\text { Au oxide } \\
\text { reduction } \\
\text { charge in } \mathrm{CV} \\
\text { (after Ost.) } \\
\text { (C) }\end{array}$ & $\begin{array}{l}\text { Au stripping } \\
\text { charge in } \\
\text { ASV } \\
\text { (total } \\
\text { volume) } \\
\text { (C) }\end{array}$ & $\begin{array}{c}\text { Surface } \\
\text { area (SA)/ } \\
\text { Volume } \\
\text { (after Ost.) } \\
\text { (V) }\end{array}$ & $\begin{array}{l}\text { Calculated } \\
\text { NP } \\
\text { diameter } \\
\text { based } \\
\text { on cal. } \\
\text { curve } \\
(\mathrm{nm})\end{array}$ & $\begin{array}{c}\text { Average } \\
\text { diameter of } \\
\text { NPs based } \\
\text { on cal. } \\
\text { curve } \\
\text { (nm) }\end{array}$ \\
\hline 1 & 0 & $9.46 \times 10^{-6}$ & & $3.80 \times 10^{-5}$ & 0.248 & 12.1 & \multirow{4}{*}{$12.6 \pm 0.5$} \\
\hline 2 & 0 & $7.67 \times 10^{-6}$ & & $3.42 \times 10^{-5}$ & 0.224 & 13.2 & \\
\hline 3 & 0 & $1.02 \times 10^{-5}$ & & $4.39 \times 10^{-5}$ & 0.232 & 12.8 & \\
\hline 4 & 0 & $1.05 \times 10^{-5}$ & & $4.25 \times 10^{-5}$ & 0.247 & 12.1 & \\
\hline 1 & 35 & $7.84 \times 10^{-6}$ & $6.34 \times 10^{-6}$ & $3.36 \times 10^{-5}$ & 0.188 & 15.3 & \multirow{4}{*}{$14.9 \pm 1.3$} \\
\hline 2 & 35 & $1.01 \times 10^{-6}$ & $6.86 \times 10^{-6}$ & $4.04 \times 10^{-5}$ & 0.169 & 16.7 & \\
\hline 3 & 35 & $1.02 \times 10^{-6}$ & $6.61 \times 10^{-6}$ & $3.05 \times 10^{-5}$ & 0.216 & 13.6 & \\
\hline 4 & 35 & $9.64 \times 10^{-6}$ & $7.82 \times 10^{-6}$ & $3.79 \times 10^{-5}$ & 0.206 & 14.2 & \\
\hline 1 & 70 & $8.46 \times 10^{-6}$ & $7.03 \times 10^{-6}$ & $4.22 \times 10^{-5}$ & 0.167 & 16.8 & \multirow{4}{*}{$17.3 \pm 0.4$} \\
\hline 2 & 70 & $8.95 \times 10^{-6}$ & $5.42 \times 10^{-6}$ & $3.45 \times 10^{-5}$ & 0.157 & 17.7 & \\
\hline 3 & 70 & $9.20 \times 10^{-6}$ & $6.00 \times 10^{-6}$ & $3.68 \times 10^{-5}$ & 0.163 & 17.2 & \\
\hline 4 & 70 & $6.02 \times 10^{-6}$ & $4.66 \times 10^{-6}$ & $2.83 \times 10^{-5}$ & 0.164 & 17.3 & \\
\hline 1 & 105 & $8.65 \times 10^{-6}$ & $5.17 \times 10^{-6}$ & $3.81 \times 10^{-5}$ & 0.136 & 19.9 & \multirow{4}{*}{$18.9 \pm 0.8$} \\
\hline 2 & 105 & $7.67 \times 10^{-6}$ & $4.17 \times 10^{-6}$ & $2.84 \times 10^{-5}$ & 0.147 & 18.7 & \\
\hline 3 & 105 & $7.92 \times 10^{-6}$ & $4.95 \times 10^{-6}$ & $3.20 \times 10^{-5}$ & 0.155 & 17.9 & \\
\hline 4 & 105 & $7.65 \times 10^{-6}$ & $5.76 \times 10^{-6}$ & $3.96 \times 10^{-5}$ & 0.145 & 18.9 & \\
\hline 1 & 140 & $9.44 \times 10^{-6}$ & $3.84 \times 10^{-6}$ & $3.10 \times 10^{-5}$ & 0.123 & 21.5 & \multirow{4}{*}{$21.3 \pm 1.6$} \\
\hline 2 & 140 & $7.68 \times 10^{-6}$ & $2.67 \times 10^{-6}$ & $2.24 \times 10^{-5}$ & 0.119 & 22.0 & \\
\hline 3 & 140 & $7.74 \times 10^{-6}$ & $4.63 \times 10^{-6}$ & $3.20 \times 10^{-5}$ & 0.144 & 19.0 & \\
\hline 4 & 140 & $9.53 \times 10^{-6}$ & $3.45 \times 10^{-6}$ & $3.01 \times 10^{-5}$ & 0.115 & 22.6 & \\
\hline
\end{tabular}


Table S4. SA/V ratio for $15 \mathrm{~nm}$ diameter Au NPs before and after ripening at $0.45 \mathrm{~V}$.

\begin{tabular}{|c|c|c|c|c|c|c|c|}
\hline $\begin{array}{l}\text { No. } \\
\text { of } \\
\text { trial }\end{array}$ & $\begin{array}{l}\text { Time of } \\
\text { Ostwald } \\
\text { ripping } \\
\text { (min) }\end{array}$ & $\begin{array}{c}\text { Au oxide } \\
\text { reduction } \\
\text { charge in } \mathrm{CV} \\
\text { (before Ost.) } \\
\text { (C) }\end{array}$ & $\begin{array}{c}\text { Au oxide } \\
\text { reduction } \\
\text { charge in CV } \\
\text { (after Ost.) } \\
\text { (C) }\end{array}$ & $\begin{array}{l}\text { Au stripping } \\
\text { charge in ASV } \\
\text { (total volume) } \\
\text { (C) }\end{array}$ & $\begin{array}{c}\text { Surface } \\
\text { area (SA)/ } \\
\text { Volume } \\
\text { (after Ost.) } \\
\text { (V) }\end{array}$ & $\begin{array}{l}\text { Calculated } \\
\text { NP } \\
\text { diameter } \\
\text { based } \\
\text { on cal. } \\
\text { curve } \\
(\mathrm{nm})\end{array}$ & $\begin{array}{c}\text { Average } \\
\text { diameter of } \\
\text { NPs based } \\
\text { on cal. } \\
\text { curve } \\
\text { (nm) }\end{array}$ \\
\hline 1 & 0 & $9.46 \times 10^{-6}$ & & $3.80 \times 10^{-5}$ & 0.248 & 12.1 & \multirow{4}{*}{$12.6 \pm 0.5$} \\
\hline 2 & 0 & $7.67 \times 10^{-6}$ & & $3.42 \times 10^{-5}$ & 0.224 & 13.2 & \\
\hline 3 & 0 & $1.02 \times 10^{-5}$ & & $4.39 \times 10^{-5}$ & 0.232 & 12.8 & \\
\hline 4 & 0 & $1.05 \times 10^{-5}$ & & $4.25 \times 10^{-5}$ & 0.247 & 12.1 & \\
\hline 1 & 35 & $7.20 \times 10^{-6}$ & $4.22 \times 10^{-6}$ & $2.46 \times 10^{-5}$ & 0.172 & 16.5 & \multirow{4}{*}{$16.2 \pm 1.5$} \\
\hline 2 & 35 & $6.98 \times 10^{-6}$ & $5.32 \times 10^{-6}$ & $3.32 \times 10^{-5}$ & 0.160 & 17.5 & \\
\hline 3 & 35 & $7.81 \times 10^{-6}$ & $6.41 \times 10^{-6}$ & $3.08 \times 10^{-5}$ & 0.208 & 14.1 & \\
\hline 4 & 35 & $8.83 \times 10^{-6}$ & $6.06 \times 10^{-6}$ & $3.55 \times 10^{-5}$ & 0.171 & 16.6 & \\
\hline 1 & 70 & $6.75 \times 10^{-6}$ & $4.95 \times 10^{-6}$ & $3.52 \times 10^{-5}$ & 0.141 & 19.3 & \multirow{4}{*}{$19.2 \pm 0.5$} \\
\hline 2 & 70 & $7.66 \times 10^{-6}$ & $4.68 \times 10^{-6}$ & $3.42 \times 10^{-5}$ & 0.137 & 19.8 & \\
\hline 3 & 70 & $7.20 \times 10^{-6}$ & $4.98 \times 10^{-6}$ & $3.35 \times 10^{-5}$ & 0.148 & 18.6 & \\
\hline 4 & 70 & $6.16 \times 10^{-6}$ & $3.86 \times 10^{-6}$ & $2.68 \times 10^{-5}$ & 0.144 & 19.0 & \\
\hline 1 & 105 & $6.58 \times 10^{-6}$ & $3.99 \times 10^{-6}$ & $3.29 \times 10^{-5}$ & 0.121 & 21.8 & \multirow{4}{*}{$21.9 \pm 0.9$} \\
\hline 2 & 105 & $7.00 \times 10^{-6}$ & $4.18 \times 10^{-6}$ & $3.34 \times 10^{-5}$ & 0.125 & 21.2 & \\
\hline 3 & 105 & $7.24 \times 10^{-6}$ & $3.76 \times 10^{-6}$ & $3.38 \times 10^{-5}$ & 0.111 & 23.2 & \\
\hline 4 & 105 & $9.20 \times 10^{-6}$ & $4.36 \times 10^{-6}$ & $3.55 \times 10^{-5}$ & 0.123 & 21.5 & \\
\hline 1 & 140 & $8.08 \times 10^{-6}$ & $3.21 \times 10^{-6}$ & $3.71 \times 10^{-5}$ & 0.087 & 27.6 & \multirow{4}{*}{$25.5 \pm 1.4$} \\
\hline 2 & 140 & $6.52 \times 10^{-6}$ & $3.51 \times 10^{-6}$ & $3.39 \times 10^{-5}$ & 0.103 & 24.5 & \\
\hline 3 & 140 & $6.74 \times 10^{-6}$ & $2.78 \times 10^{-6}$ & $2.71 \times 10^{-5}$ & 0.102 & 24.7 & \\
\hline 4 & 140 & $8.09 \times 10^{-6}$ & $3.41 \times 10^{-6}$ & $3.42 \times 10^{-5}$ & 0.099 & 25.2 & \\
\hline
\end{tabular}


Table S5. SA/V determination of $4 \mathrm{~nm}$ diameter Au NPs after ripening at $0.0 \mathrm{~V}$.

\begin{tabular}{|c|c|c|c|c|c|c|c|}
\hline $\begin{array}{l}\text { No. } \\
\text { of } \\
\text { trial }\end{array}$ & $\begin{array}{c}\text { Time of } \\
\text { Ostwald } \\
\text { ripping } \\
\text { (min) }\end{array}$ & $\begin{array}{c}\text { Au oxide } \\
\text { reduction } \\
\text { charge in } \mathrm{CV} \\
\text { (before Ost.) } \\
\text { (C) }\end{array}$ & $\begin{array}{c}\text { Au oxide } \\
\text { reduction } \\
\text { charge in CV } \\
\text { (after Ost.) } \\
\text { (C) }\end{array}$ & $\begin{array}{l}\text { Au stripping } \\
\text { charge in ASV } \\
\text { (total volume) } \\
\text { (C) }\end{array}$ & $\begin{array}{c}\text { Surface } \\
\text { area (SA)/ } \\
\text { Volume } \\
\text { (after Ost.) } \\
\text { (V) }\end{array}$ & $\begin{array}{c}\text { Calculated } \\
\text { NP } \\
\text { diameter } \\
\text { based } \\
\text { on cal. } \\
\text { curve } \\
(\mathrm{nm})\end{array}$ & $\begin{array}{c}\text { Average } \\
\text { diameter of } \\
\text { NPs based } \\
\text { on cal. } \\
\text { curve } \\
\text { (nm) }\end{array}$ \\
\hline 1 & 0 & $2.60 \times 10^{-5}$ & & $3.77 \times 10^{-5}$ & 0.689 & 4.8 & \multirow{4}{*}{$5.1 \pm 0.2$} \\
\hline 2 & 0 & $2.35 \times 10^{-5}$ & & $3.70 \times 10^{-5}$ & 0.635 & 5.2 & \\
\hline 3 & 0 & $2.01 \times 10^{-5}$ & & $3.10 \times 10^{-5}$ & 0.648 & 5.1 & \\
\hline 4 & 0 & $3.30 \times 10^{-5}$ & & $5.08 \times 10^{-5}$ & 0.649 & 5.1 & \\
\hline 1 & 35 & $2.15 \times 10^{-5}$ & $1.94 \times 10^{-5}$ & $3.17 \times 10^{-5}$ & 0.611 & 5.4 & \multirow{4}{*}{$5.5 \pm 0.4$} \\
\hline 2 & 35 & $2.52 \times 10^{-5}$ & $2.25 \times 10^{-5}$ & $3.73 \times 10^{-5}$ & 0.603 & 6.2 & \\
\hline 3 & 35 & $2.15 \times 10^{-5}$ & $1.94 \times 10^{-5}$ & $3.17 \times 10^{-5}$ & 0.611 & 5.4 & \\
\hline 4 & 35 & $2.71 \times 10^{-5}$ & $2.47 \times 10^{-5}$ & $4.01 \times 10^{-5}$ & 0.625 & 5.2 & \\
\hline 1 & 70 & $2.65 \times 10^{-5}$ & $2.46 \times 10^{-5}$ & $4.15 \times 10^{-5}$ & 0.592 & 5.5 & \multirow{4}{*}{$5.6 \pm 0.2$} \\
\hline 2 & 70 & $2.40 \times 10^{-5}$ & $2.04 \times 10^{-5}$ & $3.40 \times 10^{-5}$ & 0.600 & 5.4 & \\
\hline 3 & 70 & $2.73 \times 10^{-5}$ & $2.54 \times 10^{-5}$ & $4.60 \times 10^{-5}$ & 0.552 & 5.9 & \\
\hline 4 & 70 & $2.61 \times 10^{-5}$ & $2.17 \times 10^{-5}$ & $3.60 \times 10^{-5}$ & 0.602 & 5.4 & \\
\hline 1 & 105 & $2.15 \times 10^{-5}$ & $2.15 \times 10^{-5}$ & $4.03 \times 10^{-5}$ & 0.533 & 6.1 & \multirow{4}{*}{$5.8 \pm 0.2$} \\
\hline 2 & 105 & $2.60 \times 10^{-5}$ & $2.30 \times 10^{-5}$ & $4.10 \times 10^{-5}$ & 0.560 & 5.8 & \\
\hline 3 & 105 & $2.77 \times 10^{-5}$ & $1,95 \times 10^{-5}$ & $3.35 \times 10^{-5}$ & 0.582 & 5.6 & \\
\hline 4 & 105 & $2.59 \times 10^{-5}$ & $2.08 \times 10^{-5}$ & $3.62 \times 10^{-5}$ & 0.574 & 5.7 & \\
\hline 1 & 140 & $2.64 \times 10^{-5}$ & $2.07 \times 10^{-5}$ & $3.92 \times 10^{-5}$ & 0.528 & 6.1 & \multirow{4}{*}{$6.4 \pm 0.4$} \\
\hline 2 & 140 & $2.72 \times 10^{-5}$ & $2.58 \times 10^{-5}$ & $4.96 \times 10^{-5}$ & 0.520 & 6.2 & \\
\hline 3 & 140 & $2.66 \times 10^{-5}$ & $2.14 \times 10^{-5}$ & $4.31 \times 10^{-5}$ & 0.518 & 6.2 & \\
\hline 4 & 140 & $1.96 \times 10^{-5}$ & $1.80 \times 10^{-5}$ & $3.94 \times 10^{-5}$ & 0.457 & 7.0 & \\
\hline
\end{tabular}


Table S6. SA/V determination of $4 \mathrm{~nm}$ diameter Au NPs after ripening at $0.30 \mathrm{~V}$.

\begin{tabular}{|c|c|c|c|c|c|c|c|}
\hline $\begin{array}{l}\text { No. } \\
\text { of } \\
\text { trial }\end{array}$ & $\begin{array}{c}\text { Time of } \\
\text { Ostwald } \\
\text { ripping } \\
\text { (min) }\end{array}$ & $\begin{array}{c}\text { Au oxide } \\
\text { reduction } \\
\text { charge in CV } \\
\text { (before Ost.) } \\
\text { (C) }\end{array}$ & $\begin{array}{c}\text { Au oxide } \\
\text { reduction } \\
\text { charge in CV } \\
\text { (after Ost.) } \\
\text { (C) }\end{array}$ & $\begin{array}{c}\text { Au stripping } \\
\text { charge in } \\
\text { ASV } \\
\text { (total volume) } \\
\text { (C) }\end{array}$ & $\begin{array}{c}\text { Surface } \\
\text { area } \\
\text { (SA)/ } \\
\text { Volume } \\
\text { (after } \\
\text { Ost.) } \\
\text { (V) } \\
\end{array}$ & $\begin{array}{l}\text { Calculated } \\
\text { NP } \\
\text { diameter } \\
\text { based } \\
\text { on cal. } \\
\text { curve } \\
(\mathrm{nm}) \\
\end{array}$ & $\begin{array}{c}\text { Average } \\
\text { diameter of } \\
\text { NPs based } \\
\text { on cal. } \\
\text { curve } \\
(\mathrm{nm})\end{array}$ \\
\hline 1 & 0 & $2.60 \times 10^{-5}$ & & $3.77 \times 10^{-5}$ & 0.689 & 4.8 & \multirow{4}{*}{$5.1 \pm 0.2$} \\
\hline 2 & 0 & $2.35 \times 10^{-5}$ & & $3.70 \times 10^{-5}$ & 0.635 & 5.2 & \\
\hline 3 & 0 & $2.01 \times 10^{-5}$ & & $3.10 \times 10^{-5}$ & 0.648 & 5.1 & \\
\hline 4 & 0 & $3.30 \times 10^{-5}$ & & $5.08 \times 10^{-5}$ & 0.649 & 5.1 & \\
\hline 1 & 35 & $2.48 \times 10^{-5}$ & $2.43 \times 10^{-5}$ & $3.93 \times 10^{-5}$ & 0.618 & 5.3 & \multirow{4}{*}{$5.7 \pm 0.3$} \\
\hline 2 & 35 & $2.30 \times 10^{-5}$ & $2.28 \times 10^{-5}$ & $4.15 \times 10^{-5}$ & 0.549 & 5.9 & \\
\hline 3 & 35 & $2.86 \times 10^{-5}$ & $2.61 \times 10^{-5}$ & $4.60 \times 10^{-5}$ & 0.567 & 5.7 & \\
\hline 4 & 35 & $2.56 \times 10^{-5}$ & $2.41 \times 10^{-5}$ & $4.27 \times 10^{-5}$ & 0.564 & 5.8 & \\
\hline 1 & 70 & $2.59 \times 10^{-5}$ & $2.10 \times 10^{-5}$ & $3.96 \times 10^{-5}$ & 0.534 & 6.1 & \multirow{4}{*}{$6.8 \pm 0.5$} \\
\hline 2 & 70 & $2.50 \times 10^{-5}$ & $1.66 \times 10^{-5}$ & $3.43 \times 10^{-5}$ & 0.483 & 6.7 & \\
\hline 3 & 70 & $1.98 \times 10^{-5}$ & $1.81 \times 10^{-5}$ & $4.03 \times 10^{-5}$ & 0.449 & 7.1 & \\
\hline 4 & 70 & $3.03 \times 10^{-5}$ & $2.58 \times 10^{-5}$ & $5.70 \times 10^{-5}$ & 0.452 & 7.1 & \\
\hline 1 & 105 & $2.21 \times 10^{-5}$ & $1.47 \times 10^{-5}$ & $4.32 \times 10^{-5}$ & 0.340 & 9.2 & \multirow{4}{*}{$8.5 \pm 0.5$} \\
\hline 2 & 105 & $2.52 \times 10^{-5}$ & $1.50 \times 10^{-5}$ & $4.23 \times 10^{-5}$ & 0.355 & 8.8 & \\
\hline 3 & 105 & $2.57 \times 10^{-5}$ & $1.58 \times 10^{-5}$ & $4.00 \times 10^{-5}$ & 0.395 & 8.0 & \\
\hline 4 & 105 & $2.63 \times 10^{-5}$ & $1.47 \times 10^{-5}$ & $3.86 \times 10^{-5}$ & 0.380 & 8.3 & \\
\hline 1 & 140 & $2.07 \times 10^{-5}$ & $1.11 \times 10^{-5}$ & $3.44 \times 10^{-5}$ & 0.322 & 9.6 & \multirow{4}{*}{$10.8 \pm 1.8$} \\
\hline 2 & 140 & $2.18 \times 10^{-5}$ & $1.10 \times 10^{-5}$ & $3.85 \times 10^{-5}$ & 0.285 & 10.7 & \\
\hline 3 & 140 & $2.38 \times 10^{-5}$ & $8.99 \times 10^{-5}$ & $4.03 \times 10^{-5}$ & 0.223 & 13.3 & \\
\hline 4 & 140 & $2.66 \times 10^{-5}$ & $1.22 \times 10^{-5}$ & $3.67 \times 10^{-5}$ & 0.332 & 9.4 & \\
\hline
\end{tabular}


Table S7. SA/V determination of $4 \mathrm{~nm}$ diameter Au NPs after ripening at $0.45 \mathrm{~V}$.

\begin{tabular}{|c|c|c|c|c|c|c|c|}
\hline $\begin{array}{l}\text { No. } \\
\text { of } \\
\text { trial }\end{array}$ & $\begin{array}{c}\text { Time of } \\
\text { Ostwald } \\
\text { ripping } \\
\text { (min) }\end{array}$ & $\begin{array}{c}\text { Au oxide } \\
\text { reduction } \\
\text { charge in } \mathrm{CV} \\
\text { (before Ost.) } \\
\text { (C) }\end{array}$ & $\begin{array}{c}\text { Au oxide } \\
\text { reduction } \\
\text { charge in } \mathrm{CV} \\
\text { (after Ost.) } \\
\text { (C) }\end{array}$ & $\begin{array}{l}\text { Au stripping } \\
\text { charge in } \\
\text { ASV } \\
\text { (total } \\
\text { volume) } \\
\text { (C) }\end{array}$ & $\begin{array}{c}\text { Surface area } \\
\text { (SA)/ } \\
\text { Volume } \\
\text { (after Ost.) } \\
\text { (V) }\end{array}$ & $\begin{array}{l}\text { Calculated } \\
\text { NP } \\
\text { diameter } \\
\text { based } \\
\text { on cal. } \\
\text { curve } \\
(\mathrm{nm})\end{array}$ & $\begin{array}{c}\text { Average } \\
\text { diameter of } \\
\text { NPs based on } \\
\text { cal. curve } \\
\text { (nm) }\end{array}$ \\
\hline 1 & 0 & $2.60 \times 10^{-5}$ & & $3.77 \times 10^{-5}$ & 0.689 & 4.8 & \multirow{4}{*}{$5.1 \pm 0.2$} \\
\hline 2 & 0 & $2.35 \times 10^{-5}$ & & $3.70 \times 10^{-5}$ & 0.635 & 5.2 & \\
\hline 3 & 0 & $2.01 \times 10^{-5}$ & & $3.10 \times 10^{-5}$ & 0.648 & 5.1 & \\
\hline 4 & 0 & $3.30 \times 10^{-5}$ & & $5.08 \times 10^{-5}$ & 0.649 & 5.1 & \\
\hline 1 & 35 & $2.90 \times 10^{-5}$ & $1.31 \times 10^{-5}$ & $3.86 \times 10^{-5}$ & 0.339 & 9.2 & \multirow{4}{*}{$9.6 \pm 0.6$} \\
\hline 2 & 35 & $2.35 \times 10^{-5}$ & $1.45 \times 10^{-6}$ & $4.13 \times 10^{-5}$ & 0.351 & 8.9 & \\
\hline 3 & 35 & $2.65 \times 10^{-5}$ & $1.21 \times 10^{-5}$ & $3.85 \times 10^{-5}$ & 0.314 & 9.9 & \\
\hline 4 & 35 & $2.71 \times 10^{-5}$ & $1.23 \times 10^{-5}$ & $4.07 \times 10^{-5}$ & 0.302 & 10.2 & \\
\hline 1 & 70 & $2.65 \times 10^{-5}$ & $1.00 \times 10^{-5}$ & $4.35 \times 10^{-5}$ & 0.229 & 13.0 & \multirow{4}{*}{$15.1 \pm 2.2$} \\
\hline 2 & 70 & $2.20 \times 10^{-5}$ & $5.14 \times 10^{-6}$ & $3.22 \times 10^{-5}$ & 0.159 & 17.6 & \\
\hline 3 & 70 & $2.68 \times 10^{-5}$ & $7.19 \times 10^{-6}$ & $4.12 \times 10^{-5}$ & 0.174 & 16.3 & \\
\hline 4 & 70 & $2.94 \times 10^{-5}$ & $1.17 \times 10^{-5}$ & $5.38 \times 10^{-5}$ & 0.217 & 13.6 & \\
\hline 1 & 105 & $2.81 \times 10^{-5}$ & $3.84 \times 10^{-6}$ & $3.10 \times 10^{-5}$ & 0.123 & 21.5 & \multirow{4}{*}{$19.3 \pm 1.6$} \\
\hline 2 & 105 & $2.77 \times 10^{-5}$ & $6.16 \times 10^{-6}$ & $3.91 \times 10^{-5}$ & 0.157 & 17.8 & \\
\hline 3 & 105 & $2.62 \times 10^{-5}$ & $5.62 \times 10^{-6}$ & $3.85 \times 10^{-5}$ & 0.146 & 18.8 & \\
\hline 4 & 105 & $2.67 \times 10^{-5}$ & $6.48 \times 10^{-6}$ & $4.52 \times 10^{-5}$ & 0.143 & 19.1 & \\
\hline 1 & 140 & $2.65 \times 10^{-5}$ & $3.15 \times 10^{-6}$ & $3.70 \times 10^{-5}$ & 0.085 & 28.1 & \multirow{4}{*}{$27.8 \pm 2.0$} \\
\hline 2 & 140 & $2.79 \times 10^{-5}$ & $4.91 \times 10^{-6}$ & $4.85 \times 10^{-5}$ & 0.101 & 24.9 & \\
\hline 3 & 140 & $2.41 \times 10^{-5}$ & $2.70 \times 10^{-6}$ & $3.44 \times 10^{-5}$ & 0.078 & 29.7 & \\
\hline 4 & 140 & $2.21 \times 10^{-5}$ & $2.00 \times 10^{-6}$ & $2.39 \times 10^{-5}$ & 0.084 & 28.3 & \\
\hline
\end{tabular}


Table S8. SA/V determination of $1.6 \mathrm{~nm}$ diameter Au NPs after ripening at $0.30 \mathrm{~V}$.

\begin{tabular}{|c|c|c|c|c|c|c|c|}
\hline $\begin{array}{l}\text { No. } \\
\text { of } \\
\text { trial }\end{array}$ & $\begin{array}{c}\text { Time of } \\
\text { Ostwald } \\
\text { ripping } \\
\text { (min) }\end{array}$ & $\begin{array}{c}\text { Au oxide } \\
\text { reduction } \\
\text { charge in } \mathrm{CV} \\
\text { (before Ost.) } \\
\text { (C) }\end{array}$ & $\begin{array}{c}\text { Au oxide } \\
\text { reduction } \\
\text { charge in } \mathrm{CV} \\
\text { (after Ost.) } \\
\text { (C) }\end{array}$ & $\begin{array}{c}\text { Au stripping } \\
\text { charge in } \\
\text { ASV } \\
\text { (total volume) } \\
\text { (C) }\end{array}$ & $\begin{array}{c}\text { Surface } \\
\text { area (SA)/ } \\
\text { Volume } \\
\text { (after Ost.) } \\
\text { (V) }\end{array}$ & $\begin{array}{c}\text { Calculated } \\
\text { NP } \\
\text { diameter } \\
\text { based } \\
\text { on cal. } \\
\text { curve } \\
\text { (nm) } \\
\end{array}$ & $\begin{array}{c}\text { Average } \\
\text { diameter of } \\
\text { NPs based } \\
\text { on cal. } \\
\text { curve } \\
(\mathrm{nm})\end{array}$ \\
\hline 1 & 0 & $9.85 \times 10^{-5}$ & & $4.35 \times 10^{-5}$ & 2.26 & 1.5 & \multirow{4}{*}{$1.5 \pm 0.1$} \\
\hline 2 & 0 & $5.54 \times 10^{-5}$ & & $2.36 \times 10^{-5}$ & 2.34 & 1.5 & \\
\hline 3 & 0 & $5.64 \times 10^{-5}$ & & $2.40 \times 10^{-5}$ & 2.35 & 1.5 & \\
\hline 4 & 0 & $1.98 \times 10^{-4}$ & & $8.11 \times 10^{-5}$ & 2.44 & 1.4 & \\
\hline 1 & 35 & & $3.55 \times 10^{-5}$ & $3.45 \times 10^{-5}$ & 1.01 & 3.3 & \multirow{4}{*}{$3.4 \pm 0.2$} \\
\hline 2 & 35 & & $3.48 \times 10^{-5}$ & $3.40 \times 10^{-5}$ & 1.02 & 3.3 & \\
\hline 3 & 35 & & $3.40 \times 10^{-5}$ & $3.67 \times 10^{-5}$ & 0.926 & 3.6 & \\
\hline 4 & 35 & & $4.60 \times 10^{-5}$ & $4.37 \times 10^{-5}$ & 1.05 & 3.2 & \\
\hline 1 & 70 & & $2.53 \times 10^{-5}$ & $3.61 \times 10^{-5}$ & 0.700 & 4.7 & \multirow{4}{*}{$4.6 \pm 0.4$} \\
\hline 2 & 70 & & $2.93 \times 10^{-5}$ & $3.65 \times 10^{-5}$ & 0.802 & 4.1 & \\
\hline 3 & 70 & & $3.59 \times 10^{-5}$ & $5.45 \times 10^{-5}$ & 0.658 & 5.0 & \\
\hline 4 & 70 & & $3.67 \times 10^{-5}$ & $4.94 \times 10^{-5}$ & 0.740 & 4.5 & \\
\hline 1 & 105 & & $2.85 \times 10^{-5}$ & $4.83 \times 10^{-5}$ & 0.590 & 5.5 & \multirow{4}{*}{$5.5 \pm 0.3$} \\
\hline 2 & 105 & & $2.20 \times 10^{-5}$ & $3.41 \times 10^{-5}$ & 0.645 & 5.1 & \\
\hline 3 & 105 & & $1.97 \times 10^{-5}$ & $3.38 \times 10^{-5}$ & 0.582 & 5.6 & \\
\hline 4 & 105 & & $1.98 \times 10^{-5}$ & $3.45 \times 10^{-5}$ & 0.573 & 5.7 & \\
\hline 1 & 140 & & $1.19 \times 10^{-5}$ & $3.73 \times 10^{-5}$ & 0.319 & 9.7 & \multirow{4}{*}{$9.6 \pm 0.8$} \\
\hline 2 & 140 & & $1.40 \times 10^{-5}$ & $3.98 \times 10^{-5}$ & 0.351 & 8.9 & \\
\hline 3 & 140 & & $1.62 \times 10^{-5}$ & $4.68 \times 10^{-5}$ & 0.346 & 9.0 & \\
\hline 4 & 140 & & $1.23 \times 10^{-5}$ & $4.25 \times 10^{-5}$ & 0.289 & 10.6 & \\
\hline
\end{tabular}


Table S9. SA/V determination of 1:3 mixture of 4:15 nm diameter Au NPs at $0.30 \mathrm{~V}$.

\begin{tabular}{|c|c|c|c|c|c|c|c|}
\hline $\begin{array}{l}\text { No. } \\
\text { of } \\
\text { trial }\end{array}$ & $\begin{array}{l}\text { Time of } \\
\text { Ostwald } \\
\text { ripping } \\
\text { (min) }\end{array}$ & $\begin{array}{c}\text { Au oxide } \\
\text { reduction } \\
\text { charge in } \mathrm{CV} \\
\text { (before Ost.) } \\
\text { (C) }\end{array}$ & $\begin{array}{l}\text { Au oxide } \\
\text { reduction } \\
\text { charge in } \mathrm{CV} \\
\text { (after Ost.) } \\
\text { (C) }\end{array}$ & $\begin{array}{l}\text { Au stripping } \\
\text { charge in ASV } \\
\text { (total volume) } \\
\text { (C) }\end{array}$ & $\begin{array}{c}\text { Surface } \\
\text { area (SA)/ } \\
\text { Volume } \\
\text { (after Ost.) } \\
\text { (V) }\end{array}$ & $\begin{array}{l}\text { Calculated } \\
\text { NP diameter } \\
\text { based } \\
\text { on cal. curve } \\
(\mathrm{nm})\end{array}$ & $\begin{array}{c}\text { Average } \\
\text { diameter of NPs } \\
\text { based on cal. } \\
\text { curve } \\
\text { (nm) }\end{array}$ \\
\hline 1 & 0 & $1.11 \times 10^{-5}$ & & $3.60 \times 10^{-5}$ & 0.308 & 10.0 & \multirow{4}{*}{$11.1 \pm 1.0$} \\
\hline 2 & 0 & $9.10 \times 10^{-6}$ & & $3.35 \times 10^{-5}$ & 0.271 & 11.2 & \\
\hline 3 & 0 & $7.45 \times 10^{-6}$ & & $2.70 \times 10^{-5}$ & 0.275 & 11.0 & \\
\hline 4 & 0 & $8.61 \times 10^{-6}$ & & $3.45 \times 10^{-5}$ & 0.249 & 12.5 & \\
\hline 1 & 35 & $7.41 \times 10^{-6}$ & $5.16 \times 10^{-6}$ & $2.73 \times 10^{-5}$ & 0.189 & 15.3 & \multirow{3}{*}{$15.4 \pm 0.6$} \\
\hline 2 & 35 & $1.01 \times 10^{-6}$ & $7.73 \times 10^{-6}$ & $3.93 \times 10^{-5}$ & 0.196 & 14.8 & \\
\hline 3 & 35 & $9.12 \times 10^{-6}$ & $6.91 \times 10^{-6}$ & $3.89 \times 10^{-5}$ & 0.178 & 16.0 & \\
\hline 1 & 70 & $7.23 \times 10^{-6}$ & $4.72 \times 10^{-6}$ & $3.35 \times 10^{-5}$ & 0.140 & 19.5 & \multirow{3}{*}{$19.2 \pm 0.3$} \\
\hline 2 & 70 & $8.61 \times 10^{-6}$ & $5.22 \times 10^{-6}$ & $3.59 \times 10^{-5}$ & 0.145 & 18.9 & \\
\hline 3 & 70 & $9.23 \times 10^{-6}$ & $5.12 \times 10^{-6}$ & $3.60 \times 10^{-5}$ & 0.142 & 19.2 & \\
\hline 1 & 105 & $9.54 \times 10^{-6}$ & $3.00 \times 10^{-6}$ & $3.21 \times 10^{-5}$ & 0.094 & 26.4 & \multirow{3}{*}{$24.3 \pm 1.9$} \\
\hline 2 & 105 & $8.63 \times 10^{-6}$ & $3.75 \times 10^{-6}$ & $3.28 \times 10^{-5}$ & 0.114 & 22.7 & \\
\hline 3 & 105 & $1.01 \times 10^{-6}$ & $3.93 \times 10^{-5}$ & $3.64 \times 10^{-5}$ & 0.107 & 23.8 & \\
\hline 1 & 140 & $8.40 \times 10^{-6}$ & $2.43 \times 10^{-6}$ & $3.23 \times 10^{-5}$ & 0.075 & 30.5 & \multirow{3}{*}{$32.5 \pm 2.0$} \\
\hline 2 & 140 & $9.50 \times 10^{-6}$ & $2.19 \times 10^{-6}$ & $3.50 \times 10^{-5}$ & 0.062 & 34.5 & \\
\hline 3 & 140 & $1.02 \times 10^{-6}$ & $2.29 \times 10^{-5}$ & $3.33 \times 10^{-5}$ & 0.068 & 32.5 & \\
\hline
\end{tabular}


Table S10. SA/V determination of lower coverage $15 \mathrm{~nm}$ diameter Au NPs at $0.30 \mathrm{~V}$.

\begin{tabular}{|c|c|c|c|c|c|c|c|c|}
\hline $\begin{array}{l}\text { No. } \\
\text { of } \\
\text { trial }\end{array}$ & $\begin{array}{c}\text { Time of } \\
\text { Ostwald } \\
\text { ripping } \\
\text { (min) }\end{array}$ & $\begin{array}{l}\text { Au oxide } \\
\text { reduction } \\
\text { charge in } \\
\text { CV (before } \\
\text { Ost.) } \\
\text { (C) }\end{array}$ & $\begin{array}{l}\text { Au oxide } \\
\text { reduction } \\
\text { charge in } \\
\text { CV (after } \\
\text { Ost.) } \\
\text { (C) }\end{array}$ & $\begin{array}{c}\text { Au } \\
\text { stripping } \\
\text { charge in } \\
\text { ASV } \\
\text { (total } \\
\text { volume) } \\
\text { (C) }\end{array}$ & $\begin{array}{c}\text { Surface } \\
\text { area } \\
\text { (SA)/ } \\
\text { Volume } \\
\text { (after } \\
\text { Ost.) } \\
\text { (V) } \\
\end{array}$ & $\begin{array}{l}\text { Calculated } \\
\text { NP } \\
\text { diameter } \\
\text { based } \\
\text { on cal. } \\
\text { curve } \\
(\mathrm{nm}) \\
\end{array}$ & $\begin{array}{l}\text { Average } \\
\text { diameter of } \\
\text { NPs based } \\
\text { on cal. } \\
\text { curve } \\
(\mathrm{nm})\end{array}$ & $\begin{array}{c}\text { Diameter } \\
\text { (final)/ } \\
\text { Diameter } \\
\text { (initial) }\end{array}$ \\
\hline 1 & 0 & $1.35 \times 10^{-6}$ & & $6.25 \times 10^{-6}$ & 0.216 & 13.6 & \multirow[b]{3}{*}{$13.4 \pm 1.0$} & \multirow[b]{3}{*}{$1.0 \pm 0.1$} \\
\hline 2 & 0 & $1.17 \times 10^{-6}$ & & $5.70 \times 10^{-6}$ & 0.205 & 14.3 & & \\
\hline 3 & 0 & $1.69 \times 10^{-6}$ & & $6.90 \times 10^{-6}$ & 0.244 & 12.3 & & \\
\hline 1 & 70 & $1.33 \times 10^{-6}$ & $1.23 \times 10^{-6}$ & $6.77 \times 10^{-6}$ & 0.181 & 15.8 & \multirow[b]{3}{*}{$15.5 \pm 0.4$} & \multirow[b]{3}{*}{$1.2 \pm 0.1$} \\
\hline 2 & 70 & $1.47 \times 10^{-6}$ & $1.29 \times 10^{-6}$ & $7.00 \times 10^{-6}$ & 0.184 & 15.6 & & \\
\hline 3 & 70 & $1.41 \times 10^{-6}$ & $1.19 \times 10^{-6}$ & $6.16 \times 10^{-6}$ & 0.193 & 15.0 & & \\
\hline 1 & 140 & $1.28 \times 10^{-6}$ & $7.44 \times 10^{-7}$ & $5.15 \times 10^{-6}$ & 0.144 & 19.0 & \multirow[b]{3}{*}{$18.1 \pm 1.0$} & \multirow[b]{3}{*}{$1.4 \pm 0.1$} \\
\hline 2 & 140 & $1.27 \times 10^{-6}$ & $1.02 \times 10^{-6}$ & $6.65 \times 10^{-6}$ & 0.153 & 18.1 & & \\
\hline 3 & 140 & $1.16 \times 10^{-6}$ & $9.20 \times 10^{-7}$ & $5.60 \times 10^{-6}$ & 0.164 & 17.1 & & \\
\hline
\end{tabular}


Table S11. SA/V determination of higher coverage $15 \mathrm{~nm}$ diameter Au NPs at $0.30 \mathrm{~V}$.

\begin{tabular}{|c|c|c|c|c|c|c|c|c|}
\hline $\begin{array}{l}\text { No. } \\
\text { of } \\
\text { trial }\end{array}$ & $\begin{array}{c}\text { Time of } \\
\text { Ostwald } \\
\text { ripping } \\
\text { (min) }\end{array}$ & $\begin{array}{l}\text { Au oxide } \\
\text { reduction } \\
\text { charge in } \\
\text { CV } \\
\text { (before } \\
\text { Ost.) } \\
\text { (C) }\end{array}$ & $\begin{array}{l}\text { Au oxide } \\
\text { reduction } \\
\text { charge in } \\
\text { CV (after } \\
\text { Ost.) } \\
\text { (C) }\end{array}$ & $\begin{array}{c}\mathrm{Au} \\
\text { stripping } \\
\text { charge in } \\
\text { ASV } \\
\text { (total } \\
\text { volume) } \\
\text { (C) }\end{array}$ & $\begin{array}{c}\text { Surface } \\
\text { area } \\
\text { (SA)/ } \\
\text { Volume } \\
\text { (after } \\
\text { Ost.) } \\
\text { (V) }\end{array}$ & $\begin{array}{l}\text { Calculated } \\
\text { NP } \\
\text { diameter } \\
\text { based } \\
\text { on cal. } \\
\text { curve } \\
(\mathrm{nm})\end{array}$ & $\begin{array}{c}\text { Average } \\
\text { diameter of } \\
\text { NPs based } \\
\text { on cal. curve } \\
\text { (nm) }\end{array}$ & $\begin{array}{l}\text { Diameter } \\
\text { (final)/ } \\
\text { Diameter } \\
\text { (initial) }\end{array}$ \\
\hline 1 & 0 & $4.20 \times 10^{-5}$ & & $2.20 \times 10^{-4}$ & 0.190 & 15.2 & \multirow{3}{*}{$16.3 \pm 1.0$} & \multirow{3}{*}{$1.0 \pm 0.1$} \\
\hline 2 & 0 & $3.96 \times 10^{-5}$ & & $2.41 \times 10^{-4}$ & 0.164 & 17.1 & & \\
\hline 3 & 0 & $3.38 \times 10^{-5}$ & & $2.00 \times 10^{-4}$ & 0.169 & 16.7 & & \\
\hline 1 & 70 & $3.40 \times 10^{-5}$ & $1.94 \times 10^{-5}$ & $2.16 \times 10^{-4}$ & 0.089 & 27.2 & \multirow{3}{*}{$27.9 \pm 1.5$} & \multirow{3}{*}{$1.7 \pm 0.1$} \\
\hline 2 & 70 & $3.16 \times 10^{-5}$ & $1.58 \times 10^{-5}$ & $2.02 \times 10^{-4}$ & 0.078 & 29.7 & & \\
\hline 3 & 70 & $3.24 \times 10^{-5}$ & $1.82 \times 10^{-5}$ & $2.01 \times 10^{-4}$ & 0.090 & 26.9 & & \\
\hline 1 & 140 & $3.44 \times 10^{-5}$ & $1.33 \times 10^{-5}$ & $2.17 \times 10^{-4}$ & 0.061 & 34.8 & \multirow{3}{*}{$35.9 \pm 1.1$} & \multirow{3}{*}{$2.2 \pm 0.2$} \\
\hline 2 & 140 & $3.46 \times 10^{-5}$ & $1.18 \times 10^{-5}$ & $2.14 \times 10^{-4}$ & 0.055 & 37.0 & & \\
\hline 3 & 140 & $4.41 \times 10^{-5}$ & $1.25 \times 10^{-5}$ & $2.12 \times 10^{-4}$ & 0.058 & 35.9 & & \\
\hline
\end{tabular}


Table S12. Scanning Electron Microscopy (SEM) measured size of various Au NPs after different ripening times and different ripening potentials.

\begin{tabular}{|c|c|c|c|c|c|}
\hline $\begin{array}{c}\text { NP } \\
\text { initial } \\
\text { diameter } \\
(\mathrm{nm})\end{array}$ & $\begin{array}{l}\text { Time of } \\
\text { ripening } \\
\text { (min) }\end{array}$ & $\begin{array}{l}\text { Microscopic } \\
\text { average size } \\
\text { of NPs after } \\
\text { ripening at } \\
0.30 \mathrm{~V}(\mathrm{~nm})\end{array}$ & $\begin{array}{c}\text { Diameter } \\
\text { (final)/ } \\
\text { Diameter } \\
\text { (initial) at } \\
0.30 \mathrm{~V}\end{array}$ & $\begin{array}{c}\text { Microscopic } \\
\text { average size } \\
\text { of NPs after } \\
\text { ripening at } \\
0.45 \mathrm{~V}(\mathrm{~nm})\end{array}$ & $\begin{array}{c}\text { Diameter } \\
\text { (final)/ } \\
\text { Diameter } \\
\text { (initial) at } \\
0.45 \mathrm{~V}\end{array}$ \\
\hline \multirow{5}{*}{1.6} & 0 & $1.6 \pm 0.4$ & $1.0 \pm 0.3$ & & \\
\hline & 35 & & & & \\
\hline & 70 & $7.2 \pm 3.6$ & $4.5 \pm 2.5$ & & \\
\hline & 105 & & & & \\
\hline & 140 & $13.4 \pm 4.5$ & $8.4 \pm 3.5$ & & \\
\hline \multirow{5}{*}{4} & 0 & $4.1 \pm 0.7$ & $1.0 \pm 0.2$ & $4.1 \pm 0.7$ & $1.0 \pm 0.2$ \\
\hline & 35 & $7.1 \pm 1.5$ & $1.7 \pm 0.5$ & $10.2 \pm 3.4$ & $2.5 \pm 0.9$ \\
\hline & 70 & $8.3 \pm 4.7$ & $2.0 \pm 1.1$ & $13.3 \pm 5.1$ & $3.2 \pm 1.3$ \\
\hline & 105 & $9.9 \pm 3.8$ & $2.4 \pm 1.0$ & $17.0 \pm 7.0$ & $4.1 \pm 1.8$ \\
\hline & 140 & $14.4 \pm 6.0$ & $3.5 \pm 1.6$ & $26.5 \pm 14$ & $6.5 \pm 3.6$ \\
\hline \multirow{5}{*}{15} & 0 & $15.1 \pm 1.6$ & $1.0 \pm 0.1$ & $15.1 \pm 1.6$ & $1.0 \pm 0.1$ \\
\hline & 35 & $16.1 \pm 2.8$ & $1.1 \pm 0.2$ & $17.9 \pm 3.2$ & $1.2 \pm 0.2$ \\
\hline & 70 & $17.6 \pm 3.7$ & $1.2 \pm 0.3$ & $19.0 \pm 4.7$ & $1.3 \pm 0.4$ \\
\hline & 105 & $19.3 \pm 4.5$ & $1.3 \pm 0.3$ & $21.3 \pm 5.1$ & $1.4 \pm 0.4$ \\
\hline & 140 & $22.0 \pm 7.5$ & $1.5 \pm 0.5$ & $26.1 \pm 7.0$ & $1.7 \pm 0.5$ \\
\hline \multirow{5}{*}{$\begin{array}{c}\text { Mix. } 4 \& \\
15\end{array}$} & 0.0 & $11.0 \pm 4.0$ & $1.0 \pm 0.5$ & & \\
\hline & 35 & $17.0 \pm 5.0$ & $1.5 \pm 0.7$ & & \\
\hline & 70 & $24.0 \pm 6.0$ & $2.2 \pm 1.0$ & & \\
\hline & 105 & $31.0 \pm 7.5$ & $2.8 \pm 1.2$ & & \\
\hline & 140 & $45.5 \pm 9.0$ & $4.1 \pm 1.7$ & & \\
\hline
\end{tabular}




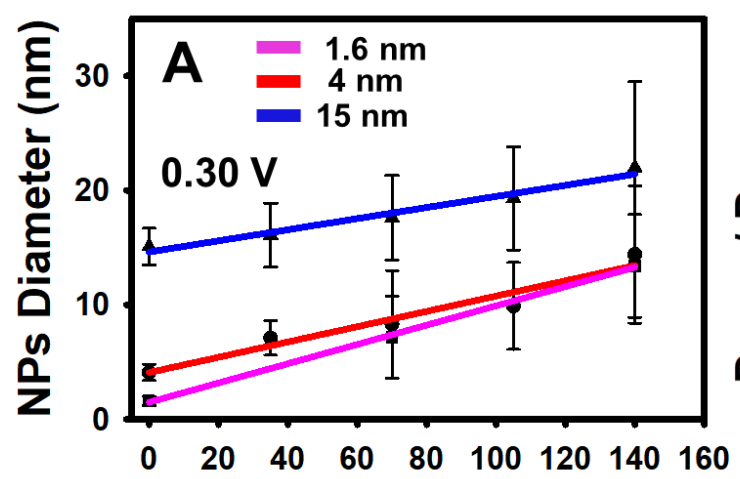

Ripening Time (min)

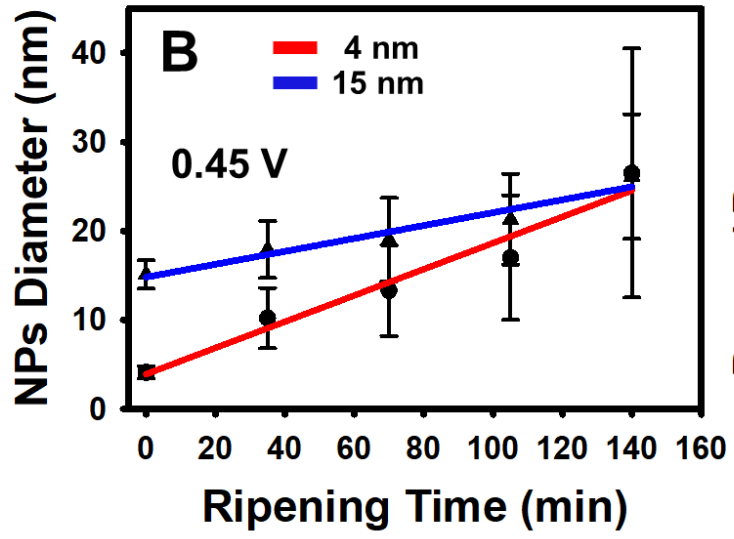

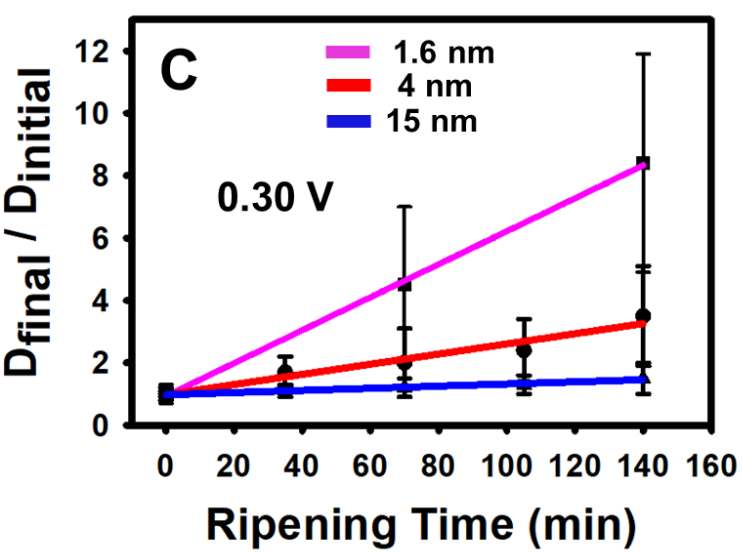

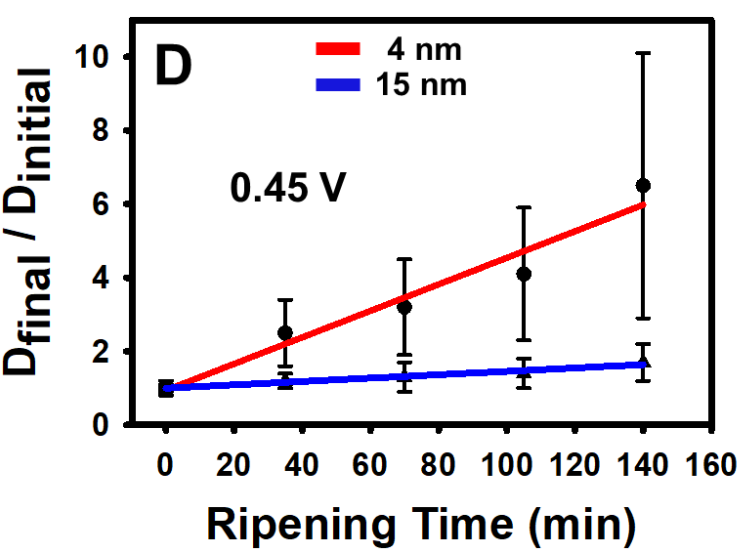

Figure S9. SEM determined NP diameter vs ripening time and $\mathrm{D}_{\text {final }} / \mathrm{D}_{\text {initial }}$ vs ripening time at $0.30 \mathrm{~V}(\mathrm{~A}, \mathrm{C})$ and $0.45 \mathrm{~V}(\mathrm{~B}, \mathrm{D})$ for all $\mathrm{Au}$ NPs samples. 

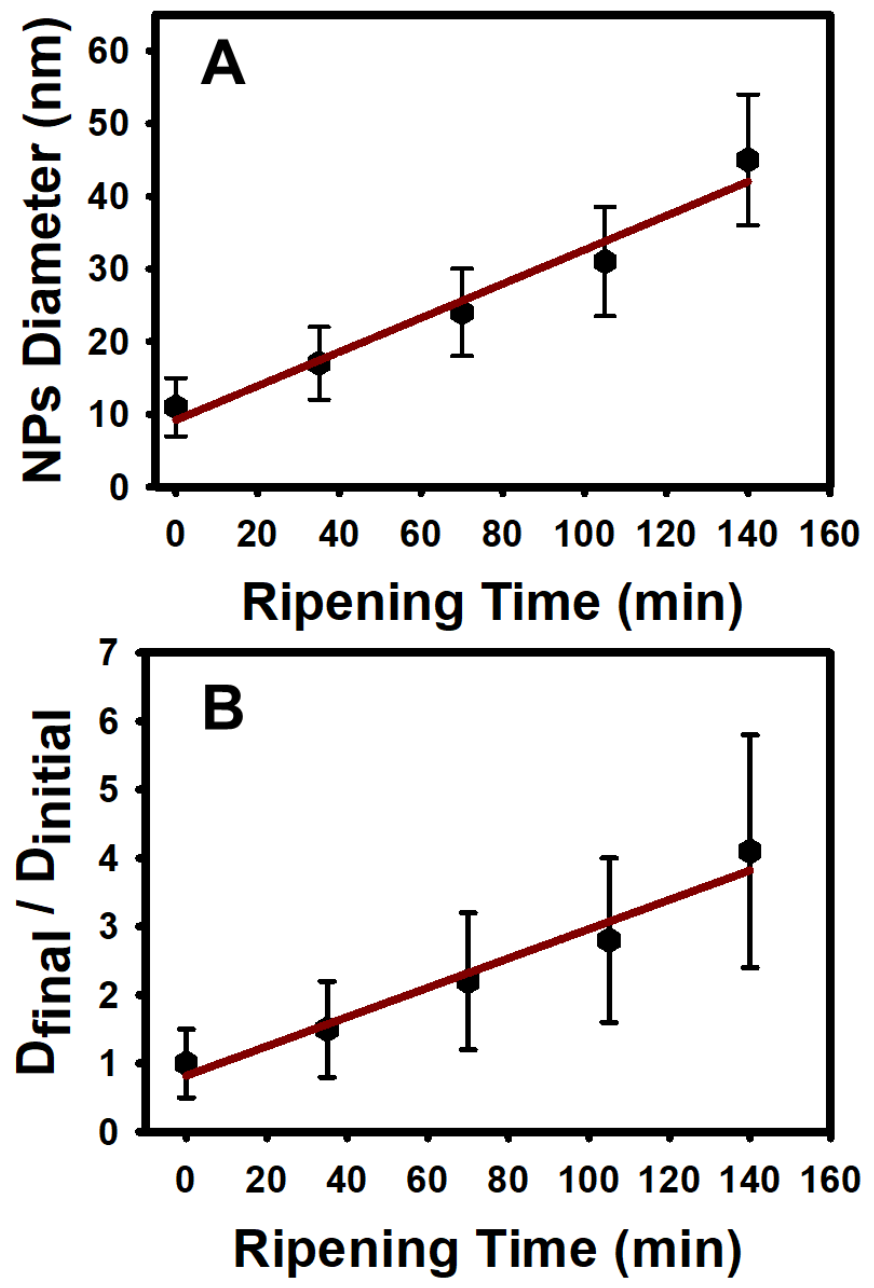

Figure S10. SEM determined NP diameter vs ripening time $(A)$ and $D_{\text {final }} / D_{\text {initial }}$ vs ripening time (B) at $0.30 \mathrm{~V}$ for 1:3 mixture of 4 and $15 \mathrm{~nm}$ diameter Au NPs samples. 

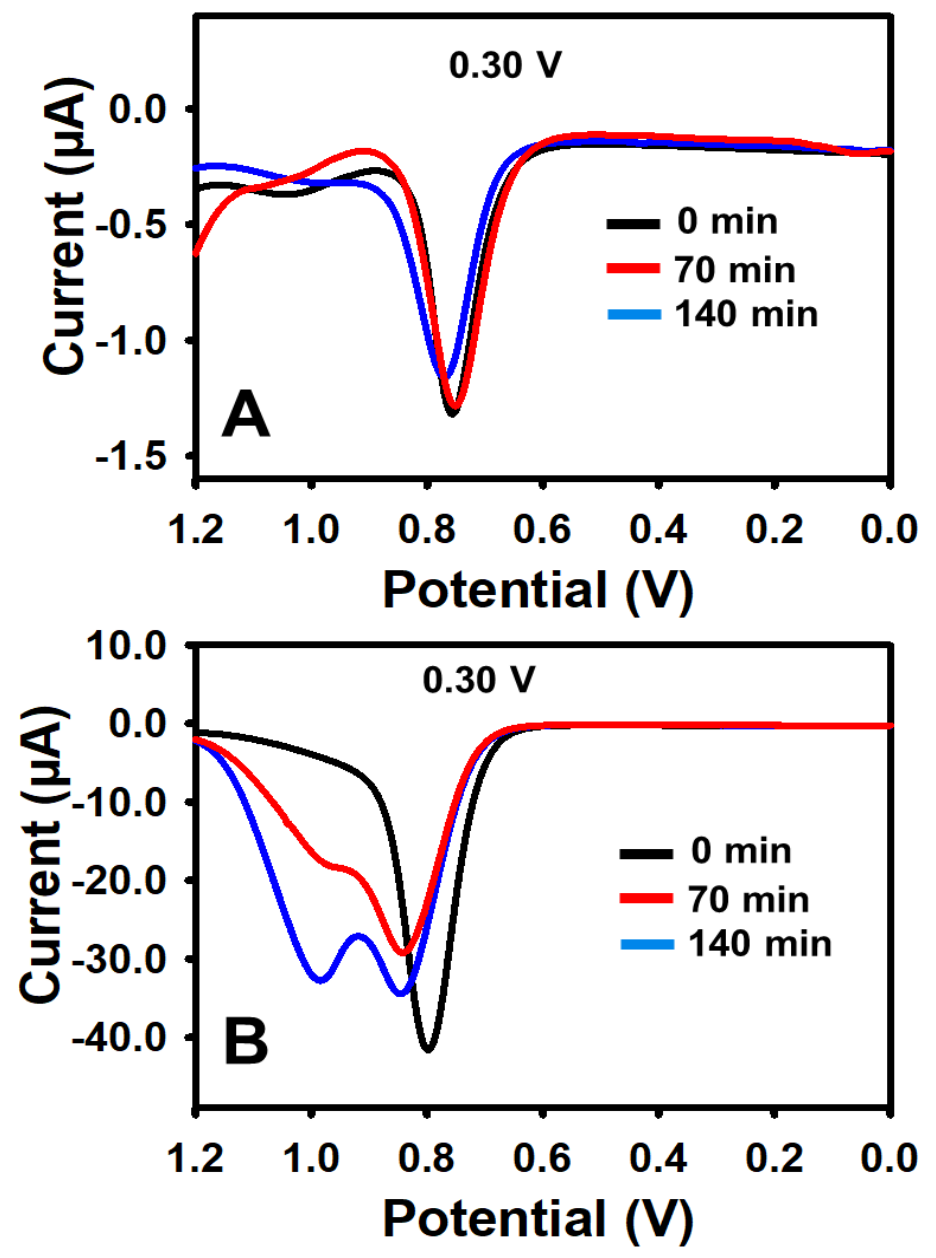

Figure S11. ASVs of citrate-stabilized $15 \mathrm{~nm}$ diameter Au NPs after Ostwald ripening at $0.30 \mathrm{~V}$ with the coverage of (A) $1.1-1.3 \mathrm{NPs} / \mu^{2}$ (or (5.0-6.5) x 10-6 C) and (B) 39.5-51.3 $\mathrm{NPs} / \mu \mathrm{m}^{2}$ (or $(2.0-2.6) \times 10^{-4} \mathrm{C}$ ). ASVs were obtained in $10 \mathrm{mM} \mathrm{KBr}$ plus $0.1 \mathrm{M} \mathrm{KClO}_{4}$ electrolyte with a scan rate of $0.01 \mathrm{~V} / \mathrm{s}$. 